\title{
A NOTE ON GENERALIZING ALTERNATIVE RINGS
}

\author{
IRVIN ROY HENTZEL ${ }^{1}$ AND GIULIA MARIA PIACENTINI CATTANEO ${ }^{2}$
}

AbSTRACT. Let $R$ be a nonassociative ring of characteristic different from 2 and 3 which satisfies the following identities:

(i) $(a b, c, d)+(a, b,[c, d])=a(b, c, d)+(a, c, d) b$,

(ii) $(a, a, a)=0$,

(iii) $(a, b \circ c, d)=b \circ(a, c, d)+c \circ(a, b, d)$

for all $a, b, c, d \in R$ and with $x \circ y=(x y+y x) / 2$. We prove that if $R$ is semiprime, then $R$ is alternative.

Introduction. In this paper we study rings $R$ which satisfy the following three identities

(i) $(a b, c, d)+(a, b,[c, d])=a(b, c, d)+(a, c, d) b$,

(ii) $(a, a, a)=0$,

(iii) $(a, b \circ c, d)=b \circ(a, c, d)+c \circ(a, b, d)$

for all $a, b, c, d \in R$. We assume characteristic different from 2 and 3 and we define the $\circ$ product by $x \circ y=(x y+y x) / 2$.

Identities (i), (ii) and (iii) hold in any alternative ring; thus these rings are generalizations of alternative rings.

Rings of this kind have been studied by Getu and Rodabaugh. In their papers they assume the existence of an idempotent and prove that with the further assumption of simplicity [1] or primeness [4], then these rings are alternative.

In our paper we do not require the existence of an idempotent, and we prove that any semiprime ring of characteristic different from 2 and 3 which satisfies (i), (ii) and (iii) is alternative.

To prove this, we make use of the result that we stated in [2] that any semiprime ring of weakly characteristic different from 2 and 3 which satisfies (i) and (ii) is right alternative.

Preliminaries. As usual, by $(a, b, c)$ we denote the associator $(a, b$, $c)=(a b) c-a(b c)$ and by $[a, b]$ the commutator $[a, b]=a b-b a$. The associators of type $(a, a, b)$ are called alternators. An ideal $I \neq 0$ is trivial if $I^{2}=0$. Rings without trivial ideals are called semiprime.

It is known that in a right alternative ring the following identities hold (see [3]):

Received by the editors April 12, 1975.

AMS (MOS) subject classifications (1970). Primary 17A30.

Key words and phrases. Alternative, semiprime.

${ }^{1}$ Partially supported by the Iowa State University Science and Humanities Research Institute.

${ }^{2}$ Written while the second author was at Iowa State University with a fellowship from the Consiglio Nazionale delle Ricerche, Italy.

(1) American Mathematical Society 1976 


$$
\begin{gathered}
\left(a, b^{2}, c\right)=(a, b, b c+c b), \\
(a, b, b c)=(a, b, c) b .
\end{gathered}
$$

We shall also make use of the following two identities

$$
\begin{gathered}
([a, b], c, d)+(a, b,[c, d])-(b, a,[c, d]) \\
=[a,(b, c, d)]-[b,(a, c, d)], \\
{[a b, c]-a[b, c]-[a, c] b-(a, b, c)+(a, c, b)-(c, a, b)=0 .}
\end{gathered}
$$

Equation (3) is an immediate consequence of (i); Equation (4) holds in any nonassociative ring.

By characteristic different from 2 and 3 we mean that the map $x \rightarrow n x$ is $1-1$ and onto for $n=2$ and $n=3$.

By weakly characteristic different from 2 and 3 we mean that the map $x \rightarrow n x$ is $1-1$ or onto for $n=2$ and $n=3$.

Main section. Throughout the paper $R$ will be a semiprime ring of characteristic different from 2 and 3, which satisfies identities (i), (ii) and (iii). In [2] we proved that semiprime rings of weakly characteristic different from 2 and 3 satisfying identities (i) and (ii) are right alternative. We shall therefore make the assumption that $R$ is also a right alternative ring throughout the rest of the paper.

LEMMA 1. In any right alternative ring, the following identity is equivalent to (iii):

$$
(a, b,[c, b])=\lfloor b,(a, b, c)]
$$

Proof.

$$
\begin{aligned}
\left(a, b^{2}, c\right) & =(a, b, b c+c b) \text { by }(1) \\
& =2(a, b, c) b+(a, b,[c, b]) \text { by }(2) \\
& =2(a, b, c) \circ b-[b,(a, b, c)]+(a, b,[c, b]) .
\end{aligned}
$$

LEMMA 2. $([a, b], a, b)=0$ for all $a, b \in R$.

Proof. From Lemma $1,(a, b,[a, b])=[b,(a, b, a)]$ and $(b, a,[b, a])=[a$, $(b, a, b)]$. Adding these gives $(a, b,[a, b])-(b, a,[a, b])=[a,(b, a, b)]-$ $[b,(a, a, b)]$. Comparing this with identity (3) when $c=a$ and $d=b$ gives $([a, b], a, b)=0$.

LEMMA 3. The additive subgroup $I$ of $R$ generated by all alternators is an ideal.

Proof. By Thedy [5, pp. 25 and 26], $I+R I$ is a left ideal which equals, if the characteristic of $R$ is different from 2 , the additive subgroup generated by all alternators and by all elements of the form $([a, b], b, a)$. Since in our rings by Lemma 2 ([a,b], $a, b)=0, I$ is a left ideal. By (3) and Lemma 1 ,

$$
([a, b], a, c)+(a, b,[a, c])=-[b,(a, a, c)] .
$$

But then, by linearizing Lemma 2 on $b$,

$$
[b,(a, a, c)]=([a, c], a, b)+(a,[a, c], b)
$$


and $I$ is also a right ideal.

Lemma 4. $(b, b,(a, a, c))=0$ for all $a, b, c \in R$.

Proof. By (5), replacing $b$ with $b^{2}$,

$$
\begin{aligned}
{\left[b^{2},(a, a, c)\right] } & =\left([a, c], a, b^{2}\right)+\left(a,[a, c], b^{2}\right) \\
= & -\left([a, c], b^{2}, a\right)-\left(a, b^{2},[a, c]\right) \\
= & -2 b \circ([a, c], b, a)-2 b \circ(a, b,[a, c]) \quad \text { by (iii) } \\
= & 2 b \circ\{([a, c], a, b)+(a,[a, c], b)\} \\
= & 2 b \circ[b,(a, a, c)] \text { by }(5) .
\end{aligned}
$$

On the other hand by (4),

$$
\left[b^{2},(a, a, c)\right]=2 b \circ[b,(a, a, c)]+2(b, b,(a, a, c)) .
$$

Subtracting the two expressions for $\left[b^{2},(a, a, c)\right]$, we get $2(b, b,(a, a, c))$ $=0$.

THEOREM. If $R$ is a semiprime ring of characteristic different from 2 and 3 and satisfies (i), (ii) and (iii), then $R$ is alternative.

Proof. Let $\lambda \in I$, where $I$ has been defined in Lemma 3. By (5) and Lemma 4,

$$
[\lambda,(a, a, b)]=([a, b], a, \lambda)+(a,[a, b], \lambda)=0 .
$$

By (iii), Lemma 3 and Lemma 4,

$$
\lambda \circ(a, a, b)=(a, a, \lambda \circ b)-(a, a, \lambda) \circ b=0 .
$$

Equations (6) and (7) yield $I^{2}=0$.

Since $R$ is semiprime, $I=0$ and $R$ is alternative.

\section{REFERENCES}

1. S. Getu and D. J. Rodabaugh, Generalizing alternative rings, Comm. Algebra 2 (1974), 35-81.

2. I. R. Hentzel and G. M. Piacentini Cattaneo, Semi-prime generalized right alternative rings, J. Algebra (to appear).

3. E. Kleinfeld, Right alternative rings, Proc. Amer. Math. Soc. 4 (1953), 939-944. MR 15, 595.

4. D. J. Rodabaugh, On generalizing alternative rings, Proc. Amer. Math. Soc. 46 (1974), 157-163.

5. A. Thedy, Right alternative rings, Preprint Series, no. 11, Matematisk Institut, Aarhus Universitet, 1972/73.

Department of Mathematics, 400 Carver Hall, Iowa State University, Ames, Iowa 50011

Istituto Matematico “G. Castelnuovo”, Universita di Roma, Rome, Italy 